\title{
CONCEPTUAL MODELLING: KNOWLEDGE ACQUISITION AND MODEL ABSTRACTION
}

\author{
Kathy Kotiadis \\ Stewart Robinson \\ Operational Research and Management Sciences Group \\ Warwick Business School \\ University of Warwick \\ Coventry, CV4 7AL, UNITED KINGDOM
}

\begin{abstract}
Conceptual modelling has gained a lot of interest in recent years and simulation modellers are particularly interested in understanding the processes involved in arriving at a conceptual model. This paper contributes to this understanding by discussing the artifacts of conceptual modelling and two specific conceptual modelling processes: knowledge acquisition and model abstraction. Knowledge acquisition is the process of finding out about the problem situation and arriving at a system description. Model abstraction refers to the simplifications made in moving from a system description to a conceptual model. Soft Systems Methodology has tools that can help a modeller with knowledge acquisition and model abstraction. These tools are drawing rich pictures, undertaking analyses 'one', 'two', 'three', and constructing a root definition and the corresponding purposeful activity model. The use of these tools is discussed with respect to a case study in health care.
\end{abstract}

\section{INTRODUCTION}

This paper focuses on two conceptual modelling processes: knowledge acquisition and model abstraction. These are explained and then the potential of Soft Systems Methodology (SSM) to help with both processes is explored.

SSM is a problem structuring approach that has previously been combined with discrete event simulation (DES) (Lehaney and Paul 1994a, 1994b; Lehaney and Hlupic 1995; Lehaney and Paul 1996; Lehaney and Paul 1999; Kotiadis 2006; Kotiadis 2007; Pidd 2007 ). However, these studies do not specifically focus on the role of SSM in knowledge acquisition and model abstraction. There is, therefore, an opportunity to reflect on SSM's contribution to the process involved in understanding the problematic situation in a rigorous and transparent fashion, and abstracting from this situation the conceptual model. Although we would argue that SSM's main contribution is to problem structuring (knowledge acquisition), it does also have a role to play in model abstraction.

The paper is divided into a further five sections. Section two provides a brief description of conceptual modelling but then focuses on the artifacts of conceptual modelling. Special attention is paid to the issues that the modeller is faced during the processes of knowledge acquisition and model abstraction. In section three SSM is briefly explained and how its various tools can be used to aid and structure the processes of knowledge acquisition and model abstraction. In section four we will discuss some of the SSM tools using an example of a health care simulation study. Finally we conclude with suggestions for future research.

\section{UNDERSTANDING CONCEPTUAL MODELLING}

In broad terms, conceptual modelling is the process of abstracting a model from the real world. The modeller is presented with a problem situation that is amenable to simulation modelling and then has to determine what aspects of the real world to include, and exclude, from the model, and at what level of detail to model each aspect. These decisions should generally be a joint agreement between the modeller and the problem owners i.e. the stakeholders who require the model to aid decision-making.

The process of conceptual modelling requires decisions to be taken regarding the scope and level of detail of the model. It also requires assumptions to be made concerning the real world and simplifications to be made to the model.

\subsection{Artifacts of Conceptual Modelling}

Figure 1 shows the key artifacts of conceptual modelling. The arrows in this diagram represent the flow of information, for instance, information about the real world feeds into the system description. The arrows are not representa- 


\section{Kotiadis and Robinson}

tive of the ordering of the steps within the modelling process, which we know are highly iterative (Balci 1994; Willemain 1995).

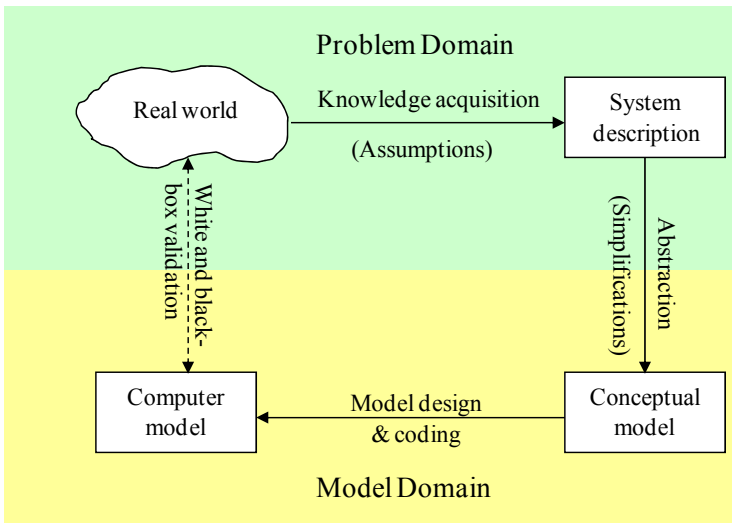

Figure 1: Artifacts of conceptual modelling

The 'cloud' represents the real world (current or future) within which the problem situation resides. The three rectangles represent specific artifacts of the (conceptual) modelling process. These are as follows:

- System description: a description of the problem situation and the system in which the problem situation resides.

- Conceptual model: "the conceptual model is a non-software specific description of the computer simulation model (that will be, is or has been developed), describing the objectives, inputs, outputs, content, assumptions and simplifications of the model.' (Robinson 2008)

- Computer model: a software specific design and software representation of the conceptual model.

These artifacts are quite separate. This is not to say that they are always explicitly expressed, with the exception of the computer model. For instance, the system description and the conceptual model may not be (fully) documented and can remain within the minds of the modeller and the problem owners. It is, of course, often good modelling practice to document both the system description and the conceptual model.

The computer model is not strictly part of conceptual modelling, but it does, of course, embody the conceptual model within the code of the model. The computer model, along with model design and coding, and white and blackbox validation, are included in Figure 1 for completeness.

It is important to recognise the distinction between the system description and the conceptual model. The system description relates to the problem domain, that is, it describes the problem and the real world. The conceptual model belongs to the model domain in that it describes what parts of the system description will be included in the simulation model and at what level of detail. The authors' experience is that these two artifacts are often confused and seen as indistinct.

The definitions here are close to those used by Zeigler (1976). The real world is equivalent to Zeigler's 'base model'; the system description to the 'experimental frame'; and the conceptual model to his 'lumped model'.

\subsection{Knowledge Acquisition and Model Abstraction in Conceptual Modelling}

The system description is obtained through knowledge acquisition. Knowledge and information about the real world is acquired from subject matter experts (SMEs). The conceptual model is obtained through abstraction. The modeller and problem owners jointly agree on what parts of the system description to model and at what level of detail. We now explore knowledge acquisition and model abstraction in some more detail.

\subsubsection{Knowledge Acquisition}

Because the real world is not fully known or knowable, the system description is only a partial representation of the real world. There are limits to the knowledge about the real world because:

- The real world has not been observed in all possible states. If the system exists, it will not have been in every state possible and so cannot have been observed in every state. In many cases the system does not yet exist; the only state in which it has been observed may be a design drawing.

- Observations about the real world are incomplete. Observers will not have been able to record every aspect of the state of the system, and often such information is very limited.

- Observations are subject to observer perceptions. Different observers may interpret events in a system differently. Hence there may be multiple accounts of the same phenomenon.

Further to this, the nature of the problem situation implies that there are a limited set of modelling objectives. It is desirable to develop a model that only addresses those objectives, rather than a general model of the system. This saves time and reduces data requirements. Hence, the system description (and conceptual model) need only focus on the parts of the real world that are relevant to the problem situation and the modelling objectives.

Because the real world is not fully known or knowable, assumptions must be made concerning the real world. 'Assumptions are made either when there are uncertainties or beliefs about the real world being modelled' (Robinson 2008). In general, assumptions are made by the problem owners in consultation with the modeller. 


\section{Kotiadis and Robinson}

It is good practice to document assumption and assess them for the confidence that can be placed in them and their likely impact on the performance of the real system. Critical assumptions (low confidence, high impact) can be assessed later with the model by performing sensitivity analysis.

\subsubsection{Model Abstraction}

Model abstraction is important because it is not desirable to model all that is known about the real world, even that which is relevant to the problem situation and modelling objectives. The benefits of simpler models are well documented (Innis and Rexstad 1983; Ward 1989; Salt 1993; Chwif et al. 2000; Lucas and McGunnigle 2003; Thomas and Charpentier 2005):

- Simple models can be developed faster

- Simple models are more flexible

- Simple models require less data

- Simple models run faster

- The results are easier to interpret since the structure of the model is better understood

Through abstraction, the conceptual model becomes a partial representation of the system description. This is achieved by reducing the scope of the conceptual model from that of the system description, and/or by reducing the level of detail in the conceptual model from that of the system description. Both of these imply a process of simplification. 'Simplifications are incorporated in the model to enable more rapid model development and use, and to improve transparency' of the model (Robinson 2008). The process of simplification should focus on maintaining sufficient accuracy for addressing the problem situation/modelling objectives.

In general, simplifications are made by the modeller in consultation with the problem owners. It is good practice to document all simplification and to assess them for their likely impact on the accuracy of the model. It is unlikely, of course, that high impact simplifications would be appropriate.

\subsubsection{Summary}

There are two distinct elements in conceptual modelling. Within the problem domain is the need to acquire knowledge about the real world and to derive a system description. This process entails making assumptions. Within the model domain is the need to abstract a conceptual model from the system description. Within this process, simplifications are made. These distinctions and definitions are important in understanding conceptual modelling and progressing research in this area.

\section{THE USE OF SSM IN KNOWLEDGE ACQUISITION AND MODEL ABSTRACTION}

Soft Systems Methodology (SSM) is a problem structuring approach that has a number of tools that may be able to practically assist in conceptual modelling. In section two we explored the conceptual modelling processes of knowledge acquisition and model abstraction from a theoretical point of view. In this section we will focus on how SSM can be used to aid and structure the process of knowledge acquisition, which largely maps on to Robinson's (2004; 2008) first conceptual modelling phase: developing an understanding of the problem situation. In addition SSM can also help in abstracting the conceptual model from the system description. This corresponds with Robinson's fifth conceptual modelling phase: determining the model content.

We will not focus on the output of conceptual modelling (the conceptual model) but the process itself. More specifically a number of issues have been identified in the sections on knowledge acquisition and model abstraction in which SSM is able to assist the modeller. The following section will start by looking at the SSM approach but then we will reflect on how SSM was approached in a case study in a health and social care system.

\subsection{A Summary of SSM}

SSM has been described as an organised learning system (Checkland 1999) that deals with complex and messy problematical situations. The process of inquiry into a situation can be organised as a learning system. In SSM the term system does not apply to a specific problematic area/domain (e.g. manufacturing system, health care system) but to the enquiry process itself. Checkland's (1999) four main activities version of the SSM methodology consists of the following stages:

1. Finding out about a problem situation, including culturally/politically;

2. Formulate some relevant purposeful activity models;

3. Debating the situation, using the models, seeking from that debate both:

- Changes which could improve the situation and are regarded as both desirable and (culturally) feasible, and

- The accommodations between conflicting interests which will enable action-to-improve to be taken;

4. Taking action in the situation to bring about improvement.

The processes of knowledge acquisition and model abstraction largely map on to SSM's first and second stage. Therefore we will only focus on these steps but readers in- 


\section{Kotiadis and Robinson}

terested in the other stages SSM or the methodology as a whole should consult Checkland (1999).

\subsection{Knowledge Acquisition: Finding out about a Problem Situation}

There are two main tools used to assist the modeller in finding out about the problem situation: drawing rich pictures and analyses one, two and three. Pidd (2007) provides an in depth discussion on the latter. We will initially discuss these tools in general and then we will reflect on their usefulness in the case study.

\subsubsection{Drawing Rich Pictures}

Rich pictures involve a holistic drawing of the situation of interest. The pictures do not have a specific format or language but aim to encompass the key elements of a situation. For example rich pictures can be drawn to include processes, issues, stakeholders. Therefore they can be drawn and understood by a wide range of stakeholders. For example they can be drawn by the modeller during a semistructured interview with a stakeholder or they can be drawn by a stakeholder or modeller in a participative fashion with a group of stakeholders. Therefore the advantage of using this tool to find out about the problematic situation is that it is non technical and enables a wide participation of stakeholders.

In terms of using this tool in knowledge acquisition for a simulation study, it is likely at the end of drawing a rich picture the modeller may not have a clear understanding of what should be modeled from this problematic situation, but will have a good grasp of the overall situation. This is particularly important when the modeller(s) or stakeholders are not familiar with overall situation but only a part of it. Also any initial assumptions about the situation can be brought forward through discussion and dealt with.

This SSM tool has not been particularly reported on in simulation studies. This could be attributed to the fact that almost all studies have been in health care. Drawing rich pictures is best achieved in a participative environment (including the modeller(s) and stakeholders) with a reasonable amount of time at hand to undertake the process, which is difficult to arrange with health care professionals. Another reason is that this tool would be used at the start of the modeller(s)/stakeholders collaboration and the output of this tool tends to look like a child like drawing on flip chart paper. The modeller may feel that this tool does not fit with the image often sold to the client of working towards a computer model. On the other hand, modellers that use visual interactive simulation software have the opportunity to use the animation to discuss the problematic situation of interest but are limited to what can be drawn using the package.

\subsubsection{Analyses One, Two and Three}

In addition to the use of rich pictures Checkland (1999) advocates analyses one two and three, otherwise respectively known as role analysis, social system analysis and political system analysis.

Role analysis, or analysis one, is an analysis of the intervention system which involves exploring three main roles: the role of the client (who has caused the study to take place) the role of the 'would be problem solver' (who wants to do something about the situation) and the role of the problem owner. All or some of these roles may overlap.

Social system analysis, or analysis two, is based on the notion that a social system is a continually changing interaction of three elements: roles, norms and values. Roles are social positions of importance to the problem situation that are institutionally defined or behaviorally defined. Furthermore a role is characterised by expected behavior otherwise known as norms. In addition, performance in a role will be judged according to values.

Political system analysis, or analysis three, is about understanding how power is expressed in a particular problematic situation. For example, power may be in the form of personal charisma or even membership to a particular committee.

Understanding the roles within a problem situation, typical behavior of the stakeholders and the allocation of power can mean that the modeller can manage the stakeholders during the conceptual modelling process (and the rest of the simulation intervention) and arrive at a conceptual model that is agreeable to all, desirable and feasible. All three analyses compliment each other and do not necessarily require to be undertaken in any particular order. Also the nature of some of the questions being asked should involve a certain amount of sensitivity and that can mean that some or all of this analysis may need to be done covertly (Pidd, 2007). A possible solution to this is taking advantage of the rich picture drawing session to observe stakeholders and ask leading questions as part of analysis one, two and three. This covert analysis is feasible as stakeholders can be made to feature within the drawings.

\subsection{Model Abstraction: Formulate some Relevant Purposeful Activity Models}

A purposeful activity model (PAM) is an SSM model. The initial stage to developing a PAM is to define the system of interest using a structured approach involving a set SSM of tools. Checkland (1999) provides both extensive guidance and examples of how to use the SSM tools (transformation process, CATWOE, root definition, measures of performance) to arrive at the PAM. These tools offer guidance on how to format a set of definitions to help develop the PAM. The definition of the system, called the root definition, can be loosely compared to a 


\section{Kotiadis and Robinson}

company's mission statement. However, central to the root definition is a need to demonstrate the transformation process (T) of some input to output. Essentially, the process undertaken to develop the root definition is an exercise in focusing the mind on the experimental frame prior to constructing the PAM. Checkland (1999) explains that a PAM provides an idealistic view of the elements in a system and does not represent reality as participants are asked to think outside the current bounds of what is there. This enables the participants to compare reality with the idealistic view, with the aim of reaching consensus on any feasible changes.

The process of constructing the PAM involves a certain amount of simplification as it is a record of the necessary activities to support the system's transformation of input to output. Checkland (1999) recommends listing seven activities plus or minus two. The PAM is a simplification of the system description and has the potential to be used instead of the communicative conceptual model (e.g. an Activity Cycle Diagram) (Lehaney and Paul 1994a).

\section{CASE STUDY: CONCEPTUAL MODELLING OF A HEALTH AND SOCIAL CARE SYSTEM}

The study was to evaluate a health and social care system called Intermediate Care (IC) in a locality in Kent, England, in 2000. At that time, IC services were a relatively new concept and their introduction can be attributed to the growing population of older people that in many cases were found to be inappropriately using the expensive and scarce secondary care resources (hospitalbased resources). IC in contrast to acute hospital services would focus on rapid assessment, stabilisation and treatment.

It was decided that DES modelling should be deployed as it had proved itself useful in other health care studies in evaluating resources. However, we were having difficulty deciding how to model the IC system. The actual IC system at the beginning of the study was in its development phase and interviews with individual IC employees and managers revealed that it was not particularly understood as a whole by any one person in the system and there were many conflicting interpretations. There was limited documentation for individual elements of the system and none for the system as a whole. Hence SSM was used to help with developing an understanding of the system and the conceptual model.

\subsection{Analyses One, Two, Three}

In this study analyses of the roles, social and political system started early on and continued throughout most of the study. There were several people involved in this system and as the study was over a period of about three years, there were a lot of changes that redefined each analysis. For example, some employees within the system were promoted and therefore acquired more power and so could commission extensive changes to the system. Understanding the political situation meant knowing what type of system would be feasible. From the analysis of the roles we were able to understand what action some key stakeholders were prepared to undertake within the system. This also meant knowing who to persuade to organise meetings with other stakeholders to obtain information. Undertaking the social analysis enabled a better understanding of the stakeholders within the system and enabled the modeller to align herself to the culture of this health and social care system and through interaction gain access to information and insights. Also some behavior was directly or indirectly included in the simulation model.

\subsection{Drawing Rich Pictures}

During the analyses above it became apparent that it would not be possible to observe the system as a whole because of its size, the slow pace of change in the system and its geographic dispersion. Therefore, apart from observing individual services within the system and talking individually to stakeholders it was very useful to meet concurrently with a number of stakeholders with knowledge of individual sections of the system as well as the key stakeholders with power over the entire system. In this particular case study these meetings were mostly arranged on the back of preexisting meetings, which meant that there was only limited time available and the stakeholders on many occasions were preoccupied by the business from the other meeting. In addition these meetings were scheduled weeks apart from each other so the process was slow.

We did not have the time to build up rich pictures with the dedication that one would observe in a stand alone SSM study but some ready made pictures of the processes within the system were brought along for discussion. It should be noted that these were not referred to as rich pictures but as diagrams. The stakeholders were told that we were using these to help us figure out their system. In fact these diagrams were not typical rich pictures as they did not directly include issues or stakeholders. However these did emerge and get discussed within these meetings.

In hindsight it would have been better use of time for the modeller and stakeholders if a workshop was arranged for dedicated knowledge acquisition through rich picture building on the system as a whole. This opportunity could have been identified in the political or role analysis. However this was not a failure in terms of the analyses, but a failure in recognising how these key stakeholders could be best used to the advantage of the study. The information acquired using these tools should compliment each other and support the process of knowledge acquisition. 


\section{Kotiadis and Robinson}

Despite the missed opportunity to have a dedicated knowledge acquisition workshop, as no one had an overall understanding of how the system worked, the short meetings helped structure this ill-defined problem situation. Within these meetings the modeller realised that the IC system was not working as a whole because the individual services had not yet integrated their operational functions. SSM was able to deal with this because it enabled action research to take place. More specifically, the process of action research comprises of enquiry, diagnosis, action planning, action/intervention, evaluation and learning (Hart and Bond 1995). The stakeholders, who were aware of this lack of system integration, were interested in action research, which means they were willing to take action to improve the system during the study and not just as a result of the findings of the study. Therefore, it was sensible to aim at building a simulation model of a future integrated system rather than of the current situation and use SSM to determine what was considered by the stakeholders to be a desirable and feasible future system.

\subsection{The Purposeful Activity Model}

In stand alone $\mathrm{SSM}$ it is common practice to develop several root definitions and consequently PAMs within an intervention. Only one purposeful activity model (PAM) was developed in this study and that is fairly typical when using SSM in simulation studies (Kotiadis 2007). More specifically, in this case study, a primary task approach was adopted, in which only one relevant system (rather than many subsystems) that could potentially map on to an organisational boundary was concentrated upon. The focus was on building one overall PAM because the aim was to get an overall understanding of the IC system that was agreeable to all involved in order to build the simulation model. The stakeholders were asked a number of questions about the system individually or in the group meetings (when drawing rich pictures), but for reasons such as having to explain to the many stakeholders the SSM tools and how to use them within relatively short meetings, they were not directly involved in structuring the root definition or constructing the PAM. However, a group of stakeholders that included key stakeholders (with power and influence over the others) examined the root definition and PAM, and agreed that this was the most agreeable and feasible view of how the system could work.

The mnemonic CATWOE, one of Checkland's best known SSM tools and central to the process of deriving a root definition, was used to define the Customer, Actors, Transformation process, Weltanschauung (the worldview), Ownership and Environmental constraints. The definitions in terms of the IC system according to each of Checkland's acronym guidelines is as follows:
- Customers - the victims or the beneficiaries of the transformation process - are the Older People over 65 that require rehabilitation or convalescence.

- Actors - those who will do the transformation process - are the Intermediate Care employees i.e. nurses therapists etc.

- Transformation Process - the conversion of input to output - the need to support Intermediate Care in our locality is met by designing and operating a system of strategic and operational level activities.

- Weltanschauung - the worldview which makes the Transformation process meaningful in context - a belief that these strategic and operational level activities are important in providing effective care for the older people.

- Ownership - those who could stop the transformation process - the local health and social care authorities.

- Environmental Constraints - elements outside the system which is takes as given - local IC funding, Department of Health guidelines etc.

Using Checkland's (1999) guidance on how to cast a root definition but also taking into account our CATWOE definitions the following root definition was developed for our system: 'A local health and social care owned system operated by IC staff, that supports IC in our locality by designing and operating a system of IC strategic and operational activities in order to provide effective care for the older people, whilst recognising the constraints of local IC funding and Department of Health guidelines.' The root definition describes an agreeable future view of the IC system.

The core PAM of the IC system can be seen in Figure 2. A more detailed description of how to use the SSM tools to construct the PAM and derive the simulation study objectives can be found in Kotiadis (2007). Some of the PAM shown (left hand side) is useful in determining the simulation objectives (Kotiadis 2007). The right hand section of the PAM is closest to the computer model. Therefore the PAM is a simplification of the system description, but also with further abstraction provides the model description. More specifically, the PAM includes all the main IC operational activities (simplification / reduction in the level of detail in the conceptual model from that of the system description), but also describes in a focused way what actually takes place in the computer model (reduction in the scope of the conceptual model from that of the system description). Therefore, through this level of abstraction the conceptual model can be derived from this simple representation of the IC system. 


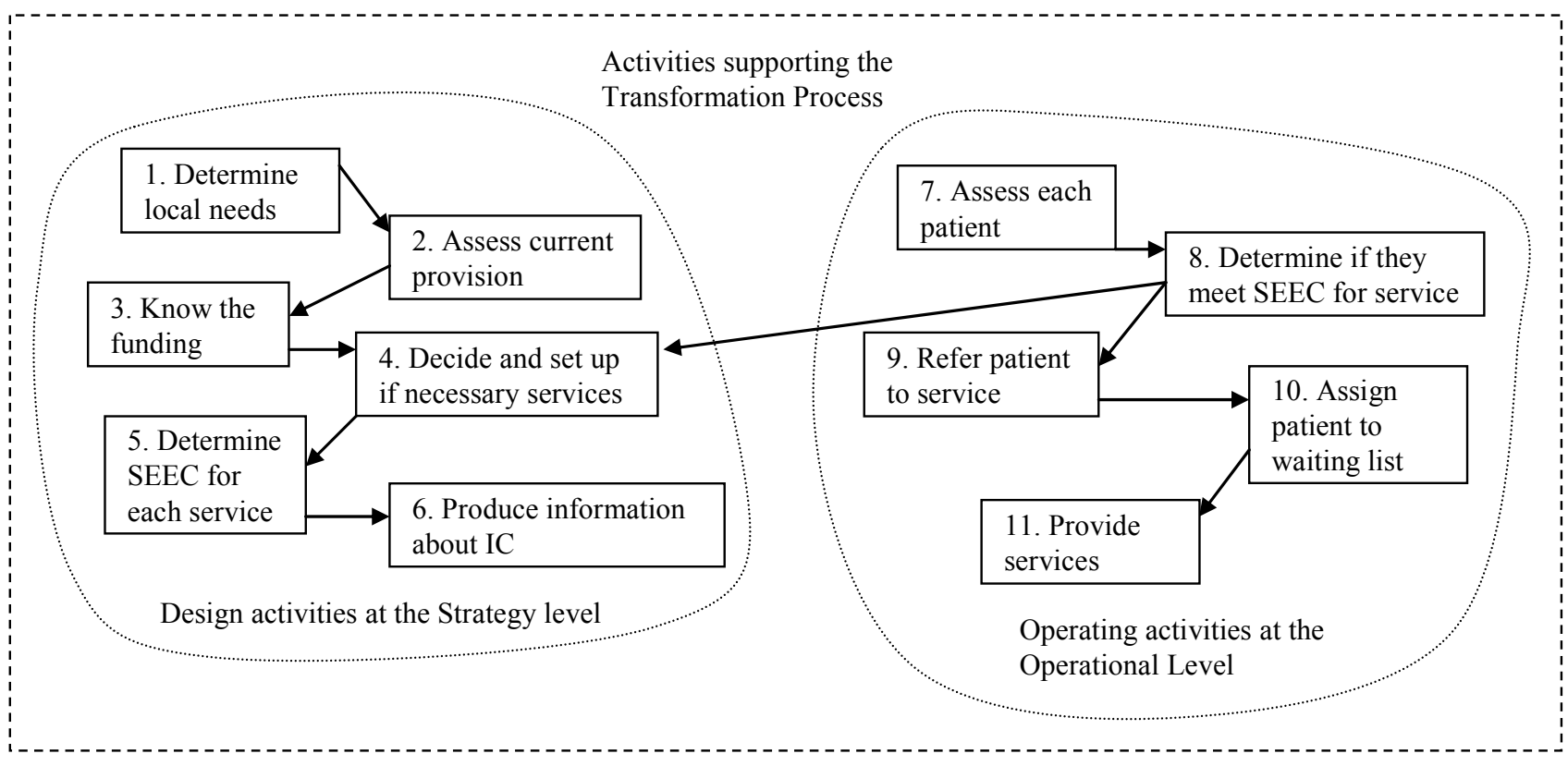

Figure 2: The core Purposeful Activity Model for the IC System

\subsection{Discussion of SSM in conceptual modelling}

This paper has focused on two conceptual modelling processes: knowledge acquisition and model abstraction. SSM, a problem structuring methodology, has been shown to be useful in knowledge acquisition and model abstraction using a case study. In this section we will continue to argue that SSM is useful by looking at two of its general benefits: it is flexible and it is a structured approach to applying common sense.

Pidd (2007) also recommends using SSM in DES to tease out various stakeholder worldviews and assumptions. He achieves this through developing more than one route definition but does not look any further at constructing the corresponding PAMs. In this paper we have focused on getting one agreeable root definition leading to one agreeable PAM. This demonstrates that SSM is not prescriptive and the SSM approach can be adapted to better map on to the specific needs of simulation studies. Checkland (1999) in his 30-year retrospective of the use of SSM provides a lengthy discussion on what constitutes a claim to using SSM, but regardless of that emphasizes that SSM should be mouldable to the situation.

It could be argued that the SSM approach is actually nothing more than common sense and much of analysis one, two and three is probably undertaken anyway by most experienced simulation modellers. However novices may find SSM helpful in undertaking their first few interventions until the process becomes internalised. Checklands (1999) puts forward two ways of thinking about the process mode 1 and mode 2. Mode 1 is a prescriptive way of thinking about the SSM process and mode 2 is internalised.

\section{CONCLUSION}

In recent years some attention has been paid to conceptual modelling in order to overcome this DES process lacuna. Process lacuna has been described as "the lack of concern in operational research (OR) with the processes that are associated with the work of analysts" (Keys 2007, p. 285). In this paper we have discussed the artifacts of conceptual modelling and focused on the processes of knowledge acquisition and model abstraction. We have argued that SSM tools can be useful in undertaking these processes and provided examples via a DES case study in health and social care. It would be useful if other studies reported their approach to knowledge acquisition and model abstraction in more detail than currently provided, regardless of whether SSM is used.

\section{REFERENCES}

Balci, O. 1994. Validation, verification, and testing techniques throughout the life cycle of a simulation study. Annals of Operations Research 53 (1): 121-173.

Checkland, P. 1999. Soft systems methodology: a 30-year retrospective. Chichester, UK: Wiley.

Chwif, L., M.R.P. Barretto, and R.J. Paul. 2000. On simulation model complexity. In Proceedings of the 2000 Winter Simulation Conference, ed. J.A. Joines, R.R. Barton, K. Kang, and P.A. Fishwick, 449-455. Pis- 


\section{Kotiadis and Robinson}

cataway, New Jersey: Institute of Electrical and Electronic Engineers, Inc.

Hart, E., and M. Bond. 1995. Action research for health and social care: a guide to practice. Buckingham, UK: Open University Press.

Innis, G., and E. Rexstad. 1983. Simulation model simplification techniques. Simulation 41 (1): 7-15.

Keys, P. 2007. Reducing the process lacuna in operational research by taking a knowledge work perspective. Systems Research and Behavioral Science 24 (3): 285-296.

Kotiadis, K. 2006. Extracting a conceptual model for a complex integrated system in health care. In Proceedings of the $3^{\text {rd }}$ Simulation Workshop (SW06), ed. J. Garnett, S. Brailsford, S. Robinson, and S. Taylor Proceedings of the OR Society's Two-Day Workshop (SW06): 235-245. Birmingham, UK: Operational Research Society.

Kotiadis, K. 2007. Using soft systems methodology to determine the simulation study objectives. Journal of Simulation 1 (3): 215-222.

Lehaney, B., S.A. Clarke, and R.J. Paul. 1999. A case of an intervention in an outpatients department. Journal of the Operational Research Society 50 (9): 877-891.

Lehaney, B., and V. Hlupic. 1995. Simulation modelling for resource allocation and planning in the health sector. Journal of the Royal Society for the Promotion of Health 115 (6): 382-385.

Lehaney, B., and R.J. Paul. 1994a. Developing sufficient conditions for an activity cycle diagram from the necessary conditions in a conceptual model. Systemist 16 (4): 262-268.

Lehaney, B., and R.J. Paul. 1994b. Using soft systems methodology to develop a simulation of outpatient services. Journal of the Royal Society for the Promotion of Health 114 (5): 248-251.

Lehaney, B., and R.J. Paul. 1996. The use of soft systems methodology in the development of a simulation of out-patients services at Watford General Hospital. Journal of the Operational Research Society 47 (7): 864-870.

Lucas, T.W., and J.E. McGunnigle. 2003. When is model complexity too much? illustrating the benefits of simple models with hughes' salvo equations. Naval Research Logistics 50 (3): 197-217.

Pidd, M. 2007 Making sure you tackle the right problem: linking hard and soft methods in simulation practice In Proceedings of the 2007 Winter Simulation Conference, ed. S.G. Henderson, B. Biller, M.-H.Hsieh, J.D.T. Shortle, and R.R. Barton, 195-204. Piscataway, New Jersey: Institute of Electrical and Electronic Engineers, Inc.

Robinson, S. 2004. Simulation: the practice of model development and use. Chichester, UK: Wiley.
Robinson, S. 2008. Conceptual modelling for simulation part I: definition and requirements. Journal of the $O p$ erational Research Society 59 (3): 278-290.

Salt, J. 1993. Simulation Should be Easy and Fun. In Proceedings of the 1993 Winter Simulation Conference, ed. G.W. Evans, M. Mollaghasemi, E.C. Russell, and W.E. Biles, 1-5. Piscataway, New Jersey: Institute of Electrical and Electronic Engineers, Inc.

Thomas, A., and Charpentier, P. 2005. Reducing simulation models for scheduling manufacturing facilities. European Journal of Operational Research 161 (1): 111-125.

Ward, S.C. 1989. Arguments for constructively simple models. Journal of the Operational Research Society 40 (2): 141-153.

Willemain, T.R. 1995. Model formulation: what experts think about and when. Operations Research 43 (6): 916-932.

Zeigler, B.P. 1976. Theory of Modeling and Simulation. New York: Wiley.

\section{AUTHOR BIOGRAPHIES}

KATHY KOTIADIS is an assistant professor at the Warwick Business School (UK) and co-chair of the UK Simulation Special Interest Group. She holds a BSc and $\mathrm{PhD}$ from the University of Kent. Her main research interests include health service modelling and combining problem structuring approaches with DESM. Her email address is <Kathy.Kotiadis@wbs.ac.uk>

STEWART ROBINSON is Professor of Operational Research and the Associate Dean for Specialist Masters Programmes at Warwick Business School. He holds a BSc and $\mathrm{PhD}$ in Management Science from Lancaster University. Previously employed in simulation consultancy, he supported the use of simulation in companies throughout Europe and the rest of the world. He is author/co-author of three books on simulation. His research focuses on the practice of simulation model development and use. Key areas of interest are conceptual modelling, model validation, output analysis and modelling human factors in simulation models. His email address is <stewart.robinson@warwick.ac.uk> and his Web address is

<wWw.btinternet. com/ stewart.robinson1/ sr.htm>. 